\title{
Simultaneidade de comportamentos de risco para a saúde e fatores associados em estudo de base populacional
}

\section{Simultaneity of health risk behaviors and associated factors in a population-based study}

\author{
Mathias Roberto Loch¹, Maira Sayuri Sakay Bortoletto², \\ Regina Kazue Tanno de Souza ${ }^{3}$, Arthur Eumann Mesas ${ }^{4}$
}

\begin{abstract}
Resumo
Objetivo: Verificar a prevalência simultânea de comportamentos relacionados à saúde e analisar a sua associação com condições sociodemográficas e de saúde na população com 40 anos ou mais de idade de um município de médio porte da região Sul do Brasil. Métodos: Trata-se de um estudo de base populacional. Os comportamentos de risco investigados foram: inatividade física no lazer, consumo irregular de frutas e de verduras, tabagismo e consumo abusivo de álcool. As variáveis dependentes do estudo foram o somatório da exposição dos comportamentos de risco e as possíveis combinações entre dois comportamentos. Resultados: Observou-se associação entre todos os comportamentos, com exceção da inatividade física no lazer com o consumo abusivo de álcool. Em geral, os homens apresentaram maior prevalência de presença de três e quatro comportamentos e entre as combinações possíveis dos comportamentos. As variáveis independentes faixa etária, nível econômico e educacional, situação conjugal e percepção de saúde também se mostraram associadas aos desfechos investigados. Conclusões: Sugere-se que as políticas e as estratégias de promoção da saúde foquem o comportamento de maneira ampla e não se limitem às ações pontuais para cada comportamento isolado.
\end{abstract}

Palavras-chave: estudos transversais; atividade física; tabagismo; abuso de álcool; alimentação.

\footnotetext{
Abstract

Objective: The aim of this study was to determine the prevalence of simultaneous health-related behaviors and examine their association with sociodemographic and health conditions in the population aged 40 or older in a medium-sized city in southern Brazil. Methods: We carried out a population-based study. The investigated risk behaviors were physical inactivity, irregular consumption of fruits and vegetables, smoking, and alcohol abuse. The dependent variables of the study were the sum of the exposure to risk behaviors and the possible two-by-two behavior combinations. Results: Associations were observed between all behaviors, except physical inactivity with alcohol abuse. In general, men presented a higher prevalence of three and four behaviors, as well as between the possible combinations of behaviors. The independent variables age, socioeconomic status, educational level, marital status, and perceived health were also associated with the investigated outcomes. Conclusions: We suggest that policies and strategies to promote health focus broadly on behavior, and not be limited to specific actions for each isolated behavior.

Keywords: cross-sectional studies; physical activity; smoking; alcohol abuse; feeding.

${ }^{1}$ Doutor em Saúde Coletiva pelo Programa de Pós-graduação em Saúde Coletiva pela Universidade Estadual de Londrina (UEL). Professor no Departamento de Educação Física da Universidade Estadual de Londrina (UEL) - Londrina (PR), Brasil.

${ }^{2}$ Doutora em Saúde Coletiva pelo Programa de Pós-graduação em Saúde Coletiva pela Universidade Estadual de Londrina (UEL). Professora no Departamento de Saúde Coletiva, Centro de Ciências da Saúde da Universidade Estadual de Londrina (UEL) - Londrina (PR), Brasil.

${ }^{3}$ Doutora em Saúde Pública pela Faculdade de Saúde Pública da Universidade de São Paulo (USP). Professora no Departamento de Saúde Coletiva, Centro de Ciências da Saúde da Universidade Estadual de Londrina (UEL) - Londrina (PR), Brasil.

${ }^{4}$ Doutor e Pós-Doutor em Medicina Preventiva e Saúde Pública pela Universidad Autónoma de Madrid. Professor no Departamento de Saúde Coletiva, Centro de Ciências da Saúde, Universidade Estadual de Londrina (UEL) - Londrina (PR), Brasil.

Trabalho realizado na Universidade Estadual de Londrina (UEL) - Londrina (PR), Brasil

Endereço para correspondência: Mathias Roberto Loch - Rod. Celso Garcia Cid PR 445 - Campus Universitário - CEP: 86051-980 - Londrina (PR), Brasil -

Email: mathiasuel@hotmail.com

Fonte de financiamento: nenhuma.

Conflito de interesses: nada a declarar.
} 


\section{INTRODUÇÃO}

A maior parte das doenças crônicas não transmissíveis está ligada a aspectos comportamentais. Fatores como tabagismo, inatividade física, dieta inadequada e consumo abusivo de álcool estão associados a importantes doenças e agravos à saúde ${ }^{1,2}$.

Muitos estudos realizam análises de cada comportamento isoladamente. Entretanto, mais recentemente, tem sido sugerida uma análise agregada, inclusive pelo entendimento de que o estilo de vida está associado a um conjunto de comportamentos. Parte-se do pressuposto que a presença de um determinado comportamento pode influenciar a presença de outros. Além disso, há de se considerar que diversos fatores exercem influência sobre várias doenças crônicas e que as mudanças para um estilo de vida saudável são normalmente indicadas como parte da prevenção e do tratamento ${ }^{3}$. Outra justificativa para o estudo agregado de comportamentos de risco é que o efeito negativo causado pela exposição simultânea tende a ser maior do que o efeito de cada comportamento isolado 4 .

O comportamento humano é determinado por uma complexa interação de variáveis e pode ser explicado por diferentes teorias e modelos. Algumas delas, como a socioecológica, destacam a existência de uma importante influência de aspectos sociais e culturais ${ }^{5}$.

Desse modo, o objetivo deste estudo foi verificar a prevalência simultânea de comportamentos relacionados à saúde e analisar a sua associação com condições sociodemográficas e de saúde na população com 40 anos ou mais de idade de um município de médio porte da região Sul do Brasil.

\section{METODOLOGIA}

Foi realizado estudo transversal com base populacional composta por moradores de 40 anos ou mais de idade, residentes na área urbana de um município de médio porte localizado na região Sul do Brasil (Cambé, no Estado do Paraná). Os dados foram coletados entre os meses de fevereiro e julho de 2011. O estudo foi aprovado previamente pelo Comitê de Ética em Pesquisa da Universidade Estadual de Londrina (UEL) (CAAE $\mathrm{n}^{\circ}$ 0192.0.268.000-10).

O tamanho da amostra foi calculado tendo por base os dados da Contagem da População 2007, época em que residiam no município de Cambé 92.888 pessoas, das quais 33,1\% tinham 40 anos ou mais de idade 6 . Utilizou-se o aplicativo StatCalc, do programa Epi Info 3.5.3, e considerou-se uma proporção esperada de $50 \%$, com margem de erro de $3 \%$ e intervalo de confiança de $95 \%$, o que resultou em uma amostra de 1.066 sujeitos. Prevendo-se eventuais perdas e recusas, foram acrescidos $25 \%$ a esse número, totalizando, inicialmente, 1.332 sujeitos a serem entrevistados. Na distribuição desse valor por sexo e por faixa etária nos setores censitários, foi necessária a realização de arredondamentos, totalizando, assim, o cálculo de 1.339 sujeitos.
Para a definição das pessoas a serem entrevistadas, foi utilizado o mapa geográfico com a divisão por setores censitários da área urbana, com bairros, ruas e quadras ${ }^{7}$. As quadras de cada setor censitário foram enumeradas e o ponto inicial do percurso foi definido por sorteio. Quando determinada a quadra que correspondia ao ponto inicial, sorteava-se o canto da quadra em que se iniciaria o percurso em sentido anti-horário. Desse modo, traçava-se aleatoriamente uma rota contígua. O domicílio inicial foi definido por sorteio, pois se optou por um intervalo amostral de 1:2, a fim de evitar concentração de entrevistados em determinadas ruas ou quadras. Quando no domicílio sorteado houvesse mais de um indivíduo que atendesse à cota por sexo e por faixa etária, a definição da pessoa a ser entrevistada foi realizada por sorteio.

Foram excluídos do estudo indivíduos que apresentassem limitações físicas ou mentais que impossibilitassem o entendimento e a execução dos procedimentos da entrevista, desde que não tivessem um cuidador apto a fornecer informações coerentes. Quando o cuidador foi o respondente, algumas perguntas que dependiam do julgamento do participante do estudo não eram realizadas.

Após a identificação do respondente, iniciava-se a apresentação do Termo de Consentimento Livre e Esclarecido (TCLE). A duração média das entrevistas foi de 30 minutos, com variação de acordo com o nível educacional e com a idade dos entrevistados. A equipe de coleta foi composta por docentes, técnico de laboratório e alunos de graduação e pós-graduação da UEL. Todos os formulários foram checados e, posteriormente, os dados foram duplamente digitados e comparados no programa Epi Info ${ }^{\circledR}$ versão 3.5.3. As inconsistências entre os dois bancos gerados foram corrigidas, a partir da conferência com os formulários originais. Para a análise dos dados, foi utilizado o programa Statistical Package for the Social Sciences ${ }^{\circledR}$ (SPSS) versão $19.0^{7}$.

No presente estudo, as variáveis comportamentais investigadas foram: fumante (consumo atual de tabaco, independentemente da quantidade), consumo abusivo de álcool (consumo de mais de quatro doses de bebidas alcoólicas para mulheres e cinco para homens, em uma mesma ocasião nos últimos 30 dias), inatividade física no lazer (não realizar nenhum tipo de atividade física no tempo livre) e consumo irregular de frutas e de verduras (consumo inferior a cinco dias na semana de frutas ou verduras).

Para avaliar a prática de atividade física no tempo livre, baseou-se no modelo de estágio de comportamento, de forma que foram considerados ativos no lazer aqueles que estavam no estágio de manutenção (faziam algum tipo de atividade física, por exemplo: caminhada, corrida, alongamento, dança ou algum tipo de atividade esportiva, no tempo de lazer há mais de seis meses) e de ação (faziam algum tipo de atividade 
física no lazer, porém há menos de seis meses). As pessoas que estavam no estágio de pré-contemplação (referiram não ter intenção em começar a praticar atividade física no lazer nos próximos seis meses), contemplação (tinham intenção de começar a praticar atividade física no lazer nos próximos seis meses) e contemplação (mencionaram ter intenção de começar a praticar atividade física no lazer nos próximos 30 dias) foram consideradas totalmente inativas no lazer8.

A variável simultaneidade foi obtida por meio do somatório da exposição dos comportamentos de risco, sendo categorizados conforme o número presente em cada sujeito investigado (nenhum, um, dois, três e quatro comportamentos). Além disso, foram criadas outras seis variáveis dependentes, conforme a combinação existente entre dois comportamentos (fumo e consumo abusivo de álcool; fumo e inatividade física no lazer; fumo e consumo irregular de frutas e de verduras; consumo abusivo de álcool e inatividade física no lazer; consumo abusivo de álcool e consumo irregular de frutas e de verduras; inatividade física no lazer e consumo irregular de frutas e de verduras).

Foram consideradas as seguintes variáveis independentes: classe econômica (conforme critério da Associação Brasileira de Empresas de Pesquisa ${ }^{9}$ ); situação conjugal (com companheiro (a) e sem companheiro(a)); anos de estudos ( $\leq 4$ anos, 5 a 8 anos e $\geq 9$ anos); faixa etária ( 40 a 49 anos, 50 a 59 anos e $\geq 60$ anos); percepção de saúde (positiva para os sujeitos que classificaram seu atual estado de saúde como muito bom ou bom; e negativa para os que referiram estado de saúde atual ser regular, ruim ou muito ruim); a presença das comorbidades hipertensão e diabetes (definida pela alteração dos níveis hemodinâmico-bioquímicos e/ou pela presença do uso de medicações para os tratamentos das respectivas doenças (quando houve o relato do uso de medicamentos, estes foram registrados mediante a apresentação da receita ou da própria medicação).

Para a análise dos dados, utilizaram-se elementos da estatística descritiva, além do teste do qui-quadrado para comparar as diferenças nas variáveis independentes entre homens e mulheres. Para a avaliação das associações entre as combinações dos comportamentos de risco (variável dependente) e as variáveis independentes, utilizou-se a técnica de regressão de Poisson com variâncias robustas, sendo ajustadas para todas as variáveis independentes. O grau de associação foi estimado pelo cálculo da razão de prevalência (RP), com os respectivos intervalos de confiança a $95 \%$ como medidas de precisão ou com o valor de $p<0,05$.

\section{RESULTADOS}

Do total de 1.336 pessoas amostradas, foram entrevistadas 1.180. Ocorreram 156 perdas $(11,7 \%)$, das quais 93 por recusa e 63 não contatadas após, no mínimo, três tentativas em horários e em dias diferentes.
Das variáveis comportamentais investigadas, a inatividade física no lazer foi o comportamento que apresentou maior prevalência $(71,4 \%)$ e foi superior entre os sujeitos com menor nível econômico e de menor escolaridade, assim como os com pior percepção de saúde. Observou-se maior prevalência de tabagistas entre os homens, entre as pessoas com menor nível econômico, entre o grupo de escolaridade intermediária (de 5 a 8 anos de estudo) e entre os indivíduos de 40 a 49 anos comparativamente aos de 60 anos e mais. Esses fatores associaram-se também ao consumo irregular de frutas e de verduras e com o consumo abusivo de álcool, exceto o nível econômico para consumo abusivo de álcool (Tabela 1).

A Tabela 2 apresenta diferenças significativas entre os sexos no somatório dos comportamentos, exceto para a categoria "dois comportamentos". A maior parte das mulheres apresentou no máximo um comportamento negativo (52,3\%), enquanto nos homens essa proporção foi de apenas $28,9 \%$. No entanto, $31,4 \%$ dos homens apresentaram três ou quatro comportamentos negativos, enquanto que $9,8 \%$ das mulheres apresentaram essa característica.

A prevalência de três ou quatro comportamentos negativos foi superior no sexo masculino, em indivíduos com menos de 60 anos, das classes econômicas mais baixas e de menor escolaridade.

A Tabela 3 apresenta a inter-relação entre as variáveis comportamentais. Com exceção da relação entre inatividade física no lazer e consumo abusivo de álcool, os demais comportamentos se associaram. A relação entre consumo abusivo de álcool e fumo apresentou a maior RP.

A análise de cada combinação entre os comportamentos com as variáveis demográficas de maneira estratificada por sexo está apresentada na Tabela 4 e na Tabela 5.

A faixa etária foi a variável independente que apresentou maior número de associações com as combinações de comportamentos analisadas. Nas mulheres, a faixa etária esteve associada a todas as combinações. Nos homens, observou-se associação da faixa etária com as seguintes variáveis: fumo com inatividade física no lazer, consumo abusivo de álcool com inatividade física no lazer, consumo abusivo de álcool com consumo irregular de frutas e de verduras e inatividade física no lazer com consumo irregular de frutas e verduras. Em todos os casos mencionados, a prevalência foi superior entre os sujeitos entre 40 e 49 anos).

O nível econômico se mostrou associado a ambos os sexos nas combinações fumo com consumo abusivo de álcool e fumo com consumo irregular de frutas e de verduras. Nas mulheres, houve também associação do consumo abusivo de álcool com a inatividade física no lazer e da inatividade física no lazer com o consumo irregular de frutas e verduras. Entre os homens, foi do fumo com a inatividade física no lazer. Em todas as combinações citadas, os sujeitos com menor nível econômico apresentaram maior prevalência nas combinações mencionadas. 
Tabela 1. Prevalência dos comportamentos relacionados à saúde, segundo variáveis sociodemográficas e de saúde e respectivos intervalos de confiança (95\%), Cambé-PR, 2011

\begin{tabular}{|c|c|c|c|c|c|}
\hline Variável & $\mathbf{N}$ & $\begin{array}{c}\text { Fumo } \\
\%(\text { IC95\%) }\end{array}$ & $\begin{array}{c}\text { Álcool } \\
\%(\text { IC95\%) }\end{array}$ & $\begin{array}{c}\text { INFL } \\
\%(\text { IC95\%) }\end{array}$ & $\begin{array}{c}\text { CIFV } \\
\%(\text { IC95\%) }\end{array}$ \\
\hline Total & $1.180^{*}$ & 20,0 & 17,5 & 71,4 & 62,5 \\
\hline \multicolumn{6}{|l|}{ Sexo } \\
\hline Masculino & 539 & $25,8(22,1-29,5)$ & $30,5(26,6-34,4)$ & $71,8(67,9-75,6)$ & $75,0(71,3-78,6)$ \\
\hline Feminino & 641 & $15,2(12,3-17,9)$ & $6,7(4,8-8,7)$ & $71,5(68,0-75,0)$ & $52,0(48,2-55,9)$ \\
\hline \multicolumn{6}{|l|}{ Nível econômico } \\
\hline $\mathrm{CDE}$ & 729 & $23,2(20,1-26,3)$ & $16,7(14,0-19,4)$ & $76,4(73,3-79,5)$ & $67,4(64,0-70,8)$ \\
\hline$A-B$ & 447 & $14,8(11,5-18,1)$ & $18,9(15,2-22,5)$ & $64,3(59,8-68,7)$ & $55,1(50,4-59,7)$ \\
\hline \multicolumn{6}{|l|}{ Escolaridade } \\
\hline$\leq 4$ anos & 566 & $19,9(16,6-23,2)$ & $12,3(9,6-15,0)$ & $77,2(73,7-80,7)$ & $65,1(61,2-69,1)$ \\
\hline 5 a 8 anos & 292 & $27,3(22,2-32,5)$ & $24,9(19,9-29,9)$ & $74,4(69,3-79,5)$ & $66,4(61,0-71,9)$ \\
\hline$\geq 9$ anos & 320 & $13,8(10,0-17,5)$ & $20,3(15,9-24,7)$ & $59,1(53,6-64,5)$ & $54,4(48,9-59,9)$ \\
\hline \multicolumn{6}{|l|}{ Faixa etária } \\
\hline$\geq 60$ anos & 335 & $14,2(10,4-18,0)$ & $7,9(4,9-10,8)$ & $68,9(63,9-73,9)$ & $54,4(49,0-59,8)$ \\
\hline 50 a 59 anos & 367 & $21,3(17,1-25,5)$ & $19,4(15,3-23,5)$ & $71,9(67,2-76,5)$ & $63,4(58,4-68,3)$ \\
\hline 40 a 49 anos & 478 & $23,1(19,3-26,9)$ & $22,9(19,1-26,7)$ & $73,3(69,3-77,3)$ & $67,4(63,2-71,7)$ \\
\hline \multicolumn{6}{|l|}{ Situação conjugal } \\
\hline Sem parceiro & 317 & $23,6(18,8-28,3)$ & $13,7(9,9-17,5)$ & $73,2(68,3-78,2)$ & $63,7(58,3-69,0)$ \\
\hline Com parceiro & 863 & $18,7(16,1-21,4)$ & $19,0(16,3-21,6)$ & $71,0(68,0-74,1)$ & $62,0(58,8-65,3)$ \\
\hline \multicolumn{6}{|l|}{ Percepção de saúde } \\
\hline Negativo & 501 & $21,6(18,0-25,2)$ & $15,2(12,0-18,4)$ & $78,8(75,2-82,4)$ & $66,4(62,2-70,6)$ \\
\hline Positivo & 672 & $18,7(15,8-21,7)$ & $19,5(16,5-22,5)$ & $66,1(62,5-69,7)$ & $59,7(55,9-63,4)$ \\
\hline \multicolumn{6}{|l|}{ Presença de HA e DM } \\
\hline Sim & 687 & $20,3(17,2-23,3)$ & $16,2(13,4-18,9)$ & $71,4(68,0-74,8)$ & $61,1(57,4-64,8)$ \\
\hline Não & 493 & $19,7(16,2-23,2)$ & $19,5(16,0-23,0)$ & $72,0(68,0-75,9)$ & $64,4(60,2-68,7)$ \\
\hline
\end{tabular}

INFL: inatividade física no lazer; CIFV: consumo irregular de frutas e verduras; ${ }^{*}$ Algumas variáveis com n menor que 1.180 em função de perdas de informações

Tabela 2. Somatório de comportamentos relacionados à saúde, segundo variáveis sociodemográficas e de saúde e respectivos intervalos de confiança (IC95\%), Cambé-PR, 2011

\begin{tabular}{|c|c|c|c|c|}
\hline \multirow{3}{*}{ Variáveis } & \multicolumn{4}{|c|}{ Somatório dos comportamentos negativos } \\
\hline & Zero & 1 & 2 & 3 ou 4 \\
\hline & $\%$ (IC95\%) & \% (IC95\%) & $\%(\mathrm{IC} 95 \%)$ & \% (IC95\%) \\
\hline Total & $11,5(9,7-13,4)$ & $30,1(27,5-33,0)$ & $38,7(35,9-41,4)$ & $19,7(18,5-22,0)$ \\
\hline \multicolumn{5}{|l|}{ Sexo } \\
\hline Feminino & $14,8(12,1-17,6)$ & $37,5(34,0-41,5)$ & $37,9(34,1-41,6)$ & $9,8(7,5-12,1)$ \\
\hline Masculino & $7,6(5,4-9,9)$ & $21,3(17,7-25,0)$ & $39,7(35,7-44,1)$ & $31,4(27,5-35,4)$ \\
\hline \multicolumn{5}{|l|}{ Faixa etária } \\
\hline$\geq 60$ anos & $14,6(11,0-18,8)$ & $37,9(33,0-43,6)$ & $37,1(31,5-42,0)$ & $10,4(6,8-13,3)$ \\
\hline 50 a 59 anos & $12,3(9,0-15,7)$ & $25,8(21,3-30,3)$ & $40,3(35,2-45,3)$ & $21,6(17,4-25,9)$ \\
\hline 40 a 49 anos & $8,2(5,8-10,8)$ & $27,8(23,8-31,9)$ & $39,1(34,7-43,4)$ & $24,9(21,0-28,8)$ \\
\hline \multicolumn{5}{|l|}{ Nível econômico } \\
\hline CDE (baixo) & $9,9(7,8-12,2)$ & $25,8(22,5-29,0)$ & $42,1(38,5-45,7)$ & $22,2(19,2-25,3)$ \\
\hline A-B (elevado) & $13,9(10,5-16,9)$ & $37,1(33,0-41,8)$ & $33,3(29,1-37,9)$ & $15,7(12,1-18,9)$ \\
\hline \multicolumn{5}{|l|}{ Escolaridade } \\
\hline$\leq 4$ anos & $10,4(7,7-12,7)$ & $29,5(26,0-33,4)$ & $40,1(36,3-44,4)$ & $20,0(16,5-23,1)$ \\
\hline 5 a 8 anos & $10,6(7,1-14,2)$ & $21,9(17,2-26,8)$ & $40,4(34,5-45,9)$ & $27,1(22,0-32,3)$ \\
\hline$\geq 9$ anos & $14,2(10,3-18,1)$ & $38,5(33,1-43,9)$ & $34,7(29,4-40,0)$ & $12,6(9,0-16,3)$ \\
\hline \multicolumn{5}{|l|}{ Situação conjugal } \\
\hline Sem parceiro & $11,0(7,5-14,50)$ & $29,1(24,0-34,2)$ & $39,5(34,0-45,0)$ & $20,4(15,9-24,9)$ \\
\hline Com parceiro & $11,5(9,4-13,7)$ & $30,6(27,4-33,6)$ & $38,5(35,3-42,0)$ & $19,4(16,8-22,1)$ \\
\hline \multicolumn{5}{|l|}{ Percepção de saúde } \\
\hline Ruim & $9,8(7,2-12,4)$ & $26,6(22,7-30,4)$ & $40,6(36,3-45,0)$ & $23,0(19,3-26,7)$ \\
\hline Boa & $12,6(10,1-15,1)$ & $32,7(29,2-36,4)$ & $37,6(33,7-41,1)$ & $17,1(14,4-20,1)$ \\
\hline \multicolumn{5}{|l|}{ Presença de HA e DM } \\
\hline Sim & $12,1(9,5-14,4)$ & $31,7(28,3-35,3)$ & $36,5(33,0-40,3)$ & $19,7(16,7-22,7)$ \\
\hline Não & $10,8(8,0-13,4)$ & $27,8(23,9-31,9)$ & $41,7(37,4-46,2)$ & $19,7(16,1-23,2)$ \\
\hline
\end{tabular}


Tabela 3. Inter-relação entre os comportamentos relacionados à saúde e respectivas razão de prevalência (RP) e intervalos de confiança (IC95\%)

\begin{tabular}{ccccc} 
& Fumo & CAA & INFL & CIFV \\
Fumo & - & - & - & - \\
CAA & $2,3(1,8-2,9)$ & - & - & - \\
INFL & $1,7(1,3-2,4)$ & $1,2(0,9-1,7)$ & - & - \\
CIFV & $1,8(1,3-2,3)$ & $2,0(1,5-2,7)$ & $1,2(1,1-1,3)$ & - \\
\hline
\end{tabular}

CAA: consumo abusivo de álcool; INFL: inatividade física no lazer; CIFV: Consumo irregular de frutas e de verduras

Tabela 4. Análise multivariada da simultaneidade dos comportamentos relacionados à saúde e associação com características sociodemográficas e de comorbidades entre as mulheres. Cambé-PR, 2011

\begin{tabular}{|c|c|c|c|c|c|c|c|c|c|c|c|c|}
\hline \multirow{2}{*}{ Variável } & \multicolumn{2}{|c|}{ Fumo e Álccol } & \multicolumn{2}{|c|}{ Fumo e INFL } & \multicolumn{2}{|c|}{ Fumo e CIFV } & \multicolumn{2}{|c|}{ Álcool e INFL } & \multicolumn{2}{|c|}{ Álcool e CIFV } & \multicolumn{2}{|c|}{ INFL e CIFV } \\
\hline & $\mathbf{R P}$ & IC95\% & RP & IC95\% & $\mathbf{R P}$ & IC95\% & $\mathbf{R P}$ & IC95\% & RP & IC95\% & RP & IC95\% \\
\hline \multicolumn{13}{|l|}{ Faixa etária } \\
\hline 40 a 49 anos & 1,0 & & 1,0 & & 1,0 & & 1,0 & & 1,0 & & 1,0 & \\
\hline 50 a 59 anos & 0,15 & $0,28-0,76$ & 0,45 & $0,25-0,79$ & 0,41 & $0,21-0,79$ & 0,29 & $0,10-0,81$ & 0,14 & $0,03-0,64$ & 0,84 & $0,67-1,04$ \\
\hline$\geq 60$ anos & $\mathrm{a}$ & & 0,22 & $0,10-0,46$ & 0,16 & $0,07-0,37$ & $\mathrm{a}$ & & $\mathrm{a}$ & & 0,61 & $0,48-0,78$ \\
\hline \multicolumn{13}{|l|}{ Nível econômico } \\
\hline A-B (elevado) & 1,0 & & 1,0 & & 1,0 & & 1,0 & & 1,0 & & 1,0 & \\
\hline CDE (baixo) & 2,87 & $1,13-7,25$ & 1,13 & $0,66-1,93$ & 2,90 & $1,27-6,62$ & 2,37 & $1,12-5,04$ & 2,72 & $0,93-7,94$ & 1,68 & $1,27-2,23$ \\
\hline \multicolumn{13}{|l|}{ Escolaridade } \\
\hline$\geq 9$ anos & 1,0 & & 1,0 & & 1,0 & & 1,0 & & 1,0 & & 1,0 & \\
\hline 5 a 8 anos & 1,05 & $0,43-2,54$ & 1,90 & $0,97-3,73$ & 1,51 & $0,68-3,35$ & 1,25 & $0,62-2,52$ & 1,27 & $0,48-3,36$ & 1,17 & $0,86-1,59$ \\
\hline$\leq 4$ anos & 0,75 & $0,26-2,16$ & 1,72 & $0,86-3,44$ & 1,56 & $0,71-3,39$ & 0,51 & $0,20-1,27$ & 0,56 & $0,19-1,68$ & 1,24 & $0,92-1,68$ \\
\hline \multicolumn{13}{|l|}{ Situação conjugal } \\
\hline Com parceiro(a) & 1,0 & & 1,0 & & 1,0 & & 1,0 & & 1,0 & & 1,0 & \\
\hline Sem parceiro(a) & 2,47 & $1,10-5,53$ & 1,43 & $0,95-2,14$ & 1,86 & $1,16-2,99$ & 2,22 & $1,18-4,18$ & 1,87 & $0,84-4,17$ & 1,14 & $0,94-1,38$ \\
\hline \multicolumn{13}{|l|}{ Percepção de saúde } \\
\hline Positiva & 1,0 & & 1,0 & & 1,0 & & 1,0 & & 1,0 & & 1,0 & \\
\hline Negativa & 1,32 & $0,59-3,00$ & 1,41 & $0,93-2,14$ & 1,21 & $0,75-1,96$ & 0,95 & $0,51-1,77$ & 1,21 & $0,57-2,57$ & 1,34 & $1,11-1,62$ \\
\hline \multicolumn{13}{|l|}{ Presença de HA/DM } \\
\hline Não & 1,0 & & 1,0 & & 1,0 & & 1,0 & & 1,0 & & 1,0 & \\
\hline Sim & 0,54 & $0,23-1,27$ & 1,18 & $0,77-1,82$ & 1,54 & $0,91-2,62$ & 0,77 & $0,41-1,49$ & 0,69 & $0,32-1,53$ & 0,97 & $0,80-1,17$ \\
\hline
\end{tabular}

INFL: inatividade física no lazer; CIFV: consumo irregular de frutas e de verduras; RP: razão de prevalência; IC: intervalo de confiança; ${ }^{a}$ : não houve casos prevalentes neste grupo etário

Tabela 5. Análise multivariada da simultaneidade dos comportamentos relacionados à saúde e associação com características sociodemográficas e de comorbidades entre os homens. Cambé-PR, 2012

\begin{tabular}{|c|c|c|c|c|c|c|c|c|c|c|c|c|}
\hline \multirow{2}{*}{ Variável } & \multicolumn{2}{|c|}{ Fumo e Álcool } & \multicolumn{2}{|c|}{ Fumo e INA } & \multicolumn{2}{|c|}{ Fumo e CIFV } & \multicolumn{2}{|c|}{ Álcool e INA } & \multicolumn{2}{|c|}{ Álcool e CIFV } & \multicolumn{2}{|c|}{ INA e CIFV } \\
\hline & RP & IC95\% & RP & IC95\% & $\mathbf{R P}$ & IC95\% & RP & IC95 & $\mathbf{R P}$ & IC95\% & $\mathbf{R P}$ & IC95\% \\
\hline \multicolumn{13}{|l|}{ Faixa etária } \\
\hline 40 a 49 anos & 1,0 & & 1,0 & & 1,0 & & 1,0 & & 1,0 & & 1,0 & \\
\hline 50 a 59 anos & 1,51 & $0,87-2,63$ & 1,10 & $0,74-1,59$ & 0,08 & $0,74-1,57$ & 0,91 & $0,64-1,28$ & 0,93 & $0,67-1,29$ & 0,76 & $0,65-0,89$ \\
\hline$\geq 60$ anos & 0,43 & $0,17-1,07$ & 0,59 & $0,36-0,98$ & 0,75 & $0,47-1,21$ & 0,30 & $0,16-0,55$ & 0,35 & $0,20-0,61$ & 0,57 & $0,46-0,70$ \\
\hline \multicolumn{13}{|l|}{ Nível econômico } \\
\hline A-B (elevado) & 1,0 & & 1,0 & & 1,0 & & 1,0 & & 1,0 & & 1,0 & \\
\hline CDE (baixo) & 2,72 & $1,44-5,13$ & 1,59 & $1,04-2,42$ & 1,95 & $1,24-3,06$ & 1,04 & $0,73-1,49$ & 1,28 & $0,91-1,80$ & 1,07 & $0,91-1,26$ \\
\hline \multicolumn{13}{|l|}{ Escolaridade } \\
\hline$\geq 9$ anos & 1,0 & & 1,0 & & 1,0 & & 1,0 & & 1,0 & & 1,0 & \\
\hline 5 a 8 anos & 1,53 & $0,68-3,45$ & 2,01 & $1,10-3,67$ & 2,62 & $1,36-5,04$ & 1,40 & $0,87-2,26$ & 1,15 & $0,77-1,73$ & 1,44 & $1,13-1,83$ \\
\hline$\leq 4$ anos & 1,31 & $0,56-3,09$ & 1,96 & $1,06-3,62$ & 2,21 & $1,09-4,45$ & 1,47 & $0,87-2,48$ & 1,01 & $0,64-1,60$ & 173 & $1,35-2,21$ \\
\hline \multicolumn{13}{|l|}{ Situação conjugal } \\
\hline Com parceiro(a) & 1,0 & & & & 1,0 & & 1,0 & & 1,0 & & 1,0 & \\
\hline Sem parceiro(a) & 0,91 & $0,48-1,73$ & 1,56 & $1,07-2,27$ & 1,71 & $1,20-2,42$ & 0,95 & $0,62-1,47$ & 0,94 & $0,62-1,41$ & 1,27 & $1,07-1,51$ \\
\hline \multicolumn{13}{|l|}{ Percepção de saúde } \\
\hline Positiva & 1,0 & & 1,0 & & 1,0 & & 1,0 & & 1,0 & & 1,0 & \\
\hline Negativa & 1,25 & $0,76-2,06$ & 1,40 & $1,01-1,94$ & 1,38 & $1,01-1,89$ & 1,46 & $1,07-1,99$ & 1,19 & $0,88-1,59$ & 1,42 & $1,24-1,63$ \\
\hline \multicolumn{13}{|l|}{ Presença de HA/DM } \\
\hline Não & 1,0 & & 1,0 & & 1,0 & & 1,0 & & 1,0 & & 1,0 & \\
\hline Sim & 1,09 & $0,67-1,77$ & 0,99 & $0,71-1,37$ & 1,09 & $0,80-1,50$ & 1,04 & $0,77-1,42$ & 0,97 & $0,73-1,30$ & 0,94 & $0,82-1,09$ \\
\hline
\end{tabular}

INA: inatividade física no lazer; CIFV = consumo irregular de frutas e de verduras; RP: razão de prevalência; IC: intervalo de confiança 
O nível de escolaridade não se mostrou associado a nenhuma combinação de comportamentos nas mulheres. Entre os homens, esteve associado ao fumo com a inatividade física no lazer, ao consumo abusivo de álcool com inatividade física e à inatividade física com o consumo irregular de frutas e de verduras. Nesses casos, os sujeitos com menor escolaridade apresentavam maior prevalência das combinações de comportamentos.

A situação conjugal se mostrou associada ao fumo com consumo irregular de frutas e de verduras em ambos os sexos. Foram ainda observadas duas associações específicas para cada sexo: nas mulheres para fumo com consumo abusivo de álcool e para consumo abusivo de álcool com inatividade física no lazer; nos homens para fumo com inatividade física no lazer e para fumo com consumo irregular de frutas e de verduras. Para as combinações mencionadas, a prevalência foi superior entre os que vivem sem parceiros.

A percepção de saúde esteve associada a ambos os sexos para inatividade física com o consumo irregular de frutas e de verduras, sendo esta a única associação encontrada, nessa variável independente, entre as mulheres. Nos homens, outras três combinações estiveram associadas: fumo com inatividade física no lazer, fumo com consumo irregular de frutas e de verduras e consumo abusivo de álcool com inatividade física no lazer. A prevalência dessas combinações foi superior entre aqueles que referiram pior percepção de saúde.

A presença de hipertensão arterial e/ou diabetes não esteve associada a nenhuma das combinações investigadas, em nenhum dos sexos.

\section{DISCUSSÃO}

Este estudo teve como objetivo verificar a prevalência simultânea de comportamentos relacionados à saúde e analisar a sua associação com condições sociodemográficas e de saúde na população com 40 anos ou mais de idade de um município de médio porte da região Sul do Brasil. Nossos achados indicaram elevada prevalência de comportamentos combinados, além de importantes associações entre as combinações investigadas e as variáveis sociodemográficas e de saúde.

Isoladamente, observou-se que, entre os comportamentos investigados, a inatividade física no lazer foi o mais prevalente, o que é condizente com outros estudos ${ }^{10,11}$. Vale mencionar que a inatividade física tem sido referida como um dos fatores mais relevantes ao risco atribuível de certos agravos, especialmente das doenças crônicas ${ }^{12}$. O fato de que, entre os comportamentos analisados, apenas a inatividade física no lazer não apresenta diferença entre os sexos reforça a influência da questão do gênero no comportamento humano ${ }^{13}$.

Apenas cerca de $10 \%$ dos sujeitos não apresentaram nenhum comportamento de risco. No entanto, a proporção de sujeitos expostos a três ou quatro comportamentos de risco foi de cerca de $20 \%$. A proporção encontrada de nenhum comportamento foi inferior a de outros estudos; já a prevalência de três ou quatro comportamentos foi superior ao encontrado na literatura. Em relação aos homens, entre os com menor nível econômico e escolaridade, assim como entre os mais jovens e os com percepção negativa de saúde, a proporção de sujeitos com três ou quatro comportamentos de risco foi superior. Apesar de algumas diferenças metodológicas, inclusive com relação aos fatores investigados, outros estudos obtiveram resultados semelhantes ${ }^{14-16}$.

Não foi observada associação entre a presença de diabetes e/ou hipertensão com o somatório dos comportamentos, sendo elevada a prevalência de sujeitos (cerca de 20\%) com pelo menos uma dessas enfermidades que apresentavam três ou quatro comportamentos de risco. Considerando os posicionamentos de órgãos internacionais propositores de políticas de controle dessas enfermidades ${ }^{17}$, a modificação desse quadro se configura como um importante desafio para a saúde pública brasileira ${ }^{18}$.

Exceto para a combinação inatividade física no lazer e consumo abusivo de álcool, todas as demais estiveram associadas de maneira significativa, reforçando que os comportamentos investigados apresentam algum tipo de inter-relação. Do mesmo modo, o contexto pode levar a associação entre outros comportamentos, por exemplo, o tabagismo e o consumo abusivo de álcool, sugerindo que pessoas preocupadas em manter ou em adotar um estilo de vida saudável tendem a planejar ações não isoladas em um dado comportamento ${ }^{3}$.

Em todas as combinações possíveis, houve maior prevalência entre os homens. Em geral, estudos que avaliam a simultaneidade de comportamentos de risco também observaram maior prevalência entre os homens ${ }^{14,16}$. Entretanto, investigação com estudantes universitários brasileiros encontrou maior proporção de três ou quatro fatores de risco comportamentais entre as mulheres ${ }^{19}$. Provavelmente essa diferença se deva às características etárias da população estudada.

De qualquer modo, há de se considerar a questão de gênero intrinsicamente envolvida nesse cenário. Parte da baixa adesão às medidas de prevenção ou de tratamento de saúde se explica por questões culturais, relacionadas inclusive aos estereótipos de gênero e pelo entendimento da doença ou da preocupação com a própria saúde como sinal de fragilidade ${ }^{20}$. Esse quadro influencia uma maior procura aos serviços de saúde por parte das mulheres ${ }^{21}$.

Observando-se os fatores associados às combinações possíveis dos comportamentos investigados, importantes associações foram encontradas em ambos os sexos. A faixa etária foi a variável independente com maior número de associações com as combinações investigadas. Em todos os casos, os sujeitos com 60 anos ou mais apresentaram menor prevalência. Em função do delineamento transversal do estudo, não é possível saber se os sujeitos com maior faixa etária modificaram o seu comportamento ou se apresentavam, quando mais jovens, o 
mesmo padrão de estilo de vida de hoje. A única informação que pode auxiliar a compreensão desse resultado se refere aos ex-fumantes. Entre os sujeitos com 60 anos ou mais, 39,4\% referiram ser ex-fumantes (dados não apresentados), o que reforça a primeira hipótese, ou seja, é possível que muitos dos sujeitos com maior idade tenham comportamentos mais saudáveis atualmente do que no passado.

Além do mais, é possível que o estilo de vida das pessoas inseridas formalmente no mercado de trabalho, que estão concentradas nas faixas etárias mais baixas, dificulte a adoção de alguns comportamentos considerados saudáveis. Todavia, a mudança comportamental com o aumento da idade e o impacto do trabalho sobre o estilo de vida necessitam de investigações específicas.

Os sujeitos com menor nível econômico apresentaram maior prevalência de comportamentos combinados. Esses dados aumentam a responsabilidade do sistema público brasileiro no enfrentamento do problema dos comportamentos de risco, já que boa parte das pessoas com baixo nível econômico é usuária exclusiva do Sistema Único de Saúde ${ }^{22}$.

Também a escolaridade se mostrou associada a importantes combinações de comportamentos investigados. Os sujeitos com menor escolaridade apresentarem maior prevalência dos comportamentos de risco, o que também está de acordo com a literatura, havendo inclusive a possibilidade de que parte dessa situação se dê função de uma maior dificuldade dessas pessoas em compreender as mensagens educativas de promoção da saúde $^{23}$. Vale mencionar que essas informações são ajustadas pelas demais variáveis demográficas e de saúde, ou seja, em parte há um efeito isolado da escolaridade, que não se explica somente pelo menor nível econômico. A relação entre não ter companheiro(a) e a maior prevalência de algumas combinações investigadas é possível que esteja associada à questão do apoio social, já que pessoas com condição afetiva estável normalmente apresentam maior nível de apoio social percebido e esta pode ser uma variável importante na adoção de comportamentos saudáveis ${ }^{24}$.

A percepção de saúde se mostrou associada em apenas uma das combinações possíveis entre as mulheres e em quatro nos homens. Nesses casos, aqueles sujeitos que percebiam sua saúde de maneira negativa apresentaram maior prevalência. A autopercepção de saúde tem sido referida como um indicador objetivo de saúde ${ }^{25}$. No presente estudo, essa relação ficou mais clara entre os homens e mostrou que aqueles com comportamentos mais positivos apresentam melhor percepção de sua própria saúde.

A presença de diabetes e/ou hipertensão não se associou a nenhuma das combinações investigadas. No entanto, sabe-se que, a prevalência de diabetes e hipertensão tende a ser superior entre os sujeitos com determinados comportamentos de risco à saúde, quando comparados aos sujeitos com comportamentos mais saudáveis. Assim, esses dados podem ser explicados parcialmente pela causalidade reversa, ou seja, algumas pessoas com doença podem ter adotado comportamentos mais saudáveis para tratarem sua condição clínica.

Considerando os múltiplos determinantes e a complexidade do comportamento humano, este estudo reforça a ideia de que, na maioria das vezes, um comportamento negativo acaba se associando a outros comportamentos. Foi elevada a proporção de sujeitos que apresentaram pelo menos dois comportamentos de risco. Levando-se em conta a relação entre os comportamentos com o quadro atual de saúde, principalmente com a elevada prevalência de doenças crônicas não transmissíveis, as políticas públicas que têm como foco a mudança do comportamento deveriam considerar essa informação. Mesmo havendo especificidades importantes em cada comportamento, sugere-se que as estratégias foquem a mudança de comportamento como um todo e não se limitem às ações pontuais para cada comportamento, estabelecendo, assim, estratégias mais amplas de promoção da saúde.

Além disso, ações específicas voltadas aos homens, aos sujeitos com menor idade, aos mais pobres e aos sem parceiros(as) podem ser importantes e deverão envolver aspectos amplos, como a modificação do imaginário dos homens e dos mais jovens com relação aos comportamentos relacionados à saúde e a criação de estratégias que aumentem a possibilidade de as pessoas com menor nível econômico adotarem comportamentos considerados positivos para a saúde.

\section{REFERÊNCIAS}

1. Schmidt MI, Duncan BB, Silva GA, Menezes AM, Monteiro CA, Barreto SM, et al. Chronic non-communicable diseases in Brazil: burden and current challenges. Lancet. 2011 Jun;377(9781):1949-61. http://dx.doi. org/10.1016/S0140-6736(11)60135-9. PMid:21561658.

2. Beaglehole R, Bonita R, Horton R, Adams C, Alleyne G, Asaria P, et al. Priority actions for the non-communicable disease crisis. Lancet. 2011 Apr;377(9775):1438-47. http://dx.doi.org/10.1016/S0140-6736(11)60393-0. PMid:21474174
3. World Health Organization. Global status report on noncommunicable diseases 2010. Geneva; 2010.

4. Sacco RL, Smith SC, Holmes D, Shurin S, Brawley O, Cazap E, et al. Accelerating progress on non-communicable diseases. Lancet. 2013 Sep;382(9895):e4-5. PMid:21933747.

5. Montaño DE, Kasprzyyk D. Theory of reasoned action, theory of planned behavior, and the integrated behavior model. In: Glanz K, Rimer BK, 
Viswanath K, editors. Health behavior and health education: theory, research, and practice. United State America: Jossey-Bass; 2008. p. 67-92.

6. Instituto Brasileiro de Geografia e Estatística. [Citado em 2009 março]. Disponível em: http://www.ibge.gov.br

7. Universidade Estadual de Londrina. Vigicardio, Projeto: "Doenças Cardiovasculares no Paraná" [Internet]. [Citado em 2012 agosto 02]. Disponível em: http://www.uel.br/pos/saudecoletiva/vigicardio/index. php2011

8. Prochaska JO, Velicer WF, Rossi JS, Goldstein MG, Marcus BH, Rakowski $\mathrm{W}$, et al. Stages of change and decisional balance for 12 problem behaviors. Health Psychol. 1994;13(1):39-46.

9. Associação Brasileira de Empresas de Pesquisa. CCEB: Critério Brasil [Internet]. [Citado em 2010 maio 03]. Disponível em: http://www.abep. org/novo/Content.aspx?SectionID $=84$

10. Muniz LC, Schneider BC, Silva IC, Matijasevich A, Santos IS. Accumulated behavioral risk factors for cardiovascular diseases in Southern Brazil. Rev Saude Publica. 2012 Jun;46(3):534-42. http://dx.doi.org/10.1590/S003489102012005000021. PMid:22450564.

11. Brasil. Ministério da Saúde. Secretaria de Vigilância em Saúde - SVS. Departamento de Análise de Situação de Saúde. Vigilância de fatores de risco e proteção para doenças crônicas por inquérito telefônico: VIGITEL 2010. Brasília; 2011.

12. Hallal PC, Andersen LB, Bull FC, Guthold R, Haskell W, Ekelund U. Global physical activity levels: surveillance progress, pitfalls, and prospects. Lancet. 2012 Jul;380(9838):247-57. http://dx.doi.org/10.1016/S0140-6736(12)606461. PMid:22818937.

13. Barreto SM, Figueiredo RC. Chronic diseases, self-perceived health status and health risk behaviors: gender differences. Rev Saude Publica. 2009 Nov;43(2 Suppl 2):38-47. http://dx.doi.org/10.1590/S0034-89102009000900006. PMid:19936497.

14. Tassitano RM, Feitosa WMN, Santiago Jr GL, Tenório MCM. Simultaneidade de comportamentos de risco a saúde e fatores associados em trabalhadores da indústria. Rev. Bras. Ativ. Fís. Saúde. 2010;15(1):42-9.

15. Fine LJ, Philogene GS, Gramling R, Coups EJ, Sinha S. Prevalence of multiple chronic disease risk factors. 2001 National Health Interview Survey. Am J Prev Med. 2004 Aug;27(2 Suppl):18-24. http://dx.doi.org/10.1016/j. amepre.2004.04.017. PMid:15275670.
16. Poortinga W. The prevalence and clustering of four major lifestyle risk factors in an English adult population. Prev Med. 2007 Feb;44(2):124-8. http://dx.doi.org/10.1016/j.ypmed.2006.10.006. PMid:17157369.

17. Strong K, Mathers C, Leeder S, Beaglehole R. Preventing chronic diseases: how many lives can we save? Lancet. 2005 Oct;366(9496):1578-82. http:// dx.doi.org/10.1016/S0140-6736(05)67341-2. PMid:16257345.

18. Fine LJ, Philogene GS, Gramling R, Coups EJ, Sinha S. Prevalence of multiple chronic disease risk factors. 2001 National Health Interview Survey. Am J Prev Med. 2004 Aug;27(2 Suppl):18-24. http://dx.doi.org/10.1016/j. amepre.2004.04.017. PMid:15275670.

19. Silva DA, Petroski EL. The simultaneous presence of health risk behaviors in freshman college students in Brazil. J Community Health. 2012 Jun;37(3):5918. http://dx.doi.org/10.1007/s10900-011-9489-9. PMid:21979565.

20. Brasil. Ministério da Saúde, Departamento da atenção Primária. Política Nacional de Atenção Integral à saúde do homem. Brasília; 2008.

21. Gomes R, Nascimento EF, Araujo FC. Why do men use health services less than women? Explanations by men with low versus higher education. Cad Saúde Pública. 2007;23(3):565-74.

22. Paim J, Travassos C, Almeida C, Bahia L, Macinko J. The Brazilian health system: history, advances, and challenges. Lancet. 2011 May;377(9779):1778 97. http://dx.doi.org/10.1016/S0140-6736(11)60054-8. PMid:21561655.

23. Santa-Helena ETD, Nemes MIB, Eluf Neto J. Fatores associados à não-adesão ao tratamento com anti-hipertensivos em pessoas atendidas em unidades de saúde da família. Cad Saude Publica. 2010 Dec;26(12):2389-98. http:// dx.doi.org/10.1590/S0102-311X2010001200017. PMid:21243233.

24. Griep RH, Chor D, Faerstein E, Werneck GL, Lopes CS. Validade de constructo de escala de apoio social do Medical Outcomes Study adaptada para o português no Estudo Pró-Saúde. Cad Saude Publica. 2005 MayJun;21(3):703-14. http://dx.doi.org/10.1590/S0102-311X2005000300004. PMid:15868028.

25. Reichert FF, Loch MR, Capilheira MF. Autopercepção de saúde em adolescentes, adultos e idosos. Cien Saude Colet. 2012; 17(12):3353-62.

Recebido em: Mar. 11, 2015 Aprovado em: Jun. 10, 2015 